\title{
An Assessment of Different Optimization Strategies for Location Tracking with an Android Application on a Smartphone
}

\author{
David Plets $^{1}$, Alexander Sels, Jens Trogh ${ }^{1}$, Kris Vanhecke ${ }^{1}$, Luc Martens ${ }^{1}$, Wout Joseph ${ }^{1}$ \\ ${ }^{1}$ iMinds-INTEC/WAVES, Ghent University, Ghent, Belgium \\ *david.plets@intec.ugent.be
}

\begin{abstract}
This paper presents a study of the efficacy of different optimization strategies for location tracking on an Android App that is run on a smartphone. The basic algorithm determines the most probable path of the user within a WiFi network by comparing raw RSSI measurements at each location with values in a fingerprint database. The investigated optimization strategies include: accounting for previous locations, increasing the number of WiFi scans per location, applying an advanced averaging technique, exploiting accelerometer data, shifting the frequency band from 2.4 to $5 \mathrm{GHz}$, and changing the position of the smartphone with respect to the body. It is shown that especially the accelerometer data allow enhancing the location estimation significantly. By combining different techniques, an average accuracy better than $2 \mathrm{~m}$ can be achieved.
\end{abstract}

Index Terms-Location, location tracking, localisation, tracking, Android, Viterbi, accelerometer, sensor fusion, WiFi, RSSI, body, Android, App, wireless network, indoor, indoor environment, optimization.

\section{INTRODUCTION}

Indoor localization and tracking systems have gained a huge interest, thanks to the many domains in which they can be applied, e.g., in industrial and office environments, in the healthcare or cultural sector,... One possible application is the guidance of people towards their destination in a building, e.g., in a hospital. Such 'indoor GPS' can be deployed in many different ways: using Ultra-Wide-Band (UWB) time-of-arrival (ToA)-based localisation [1], Angle-of-Arrival (AoA)-based localisation [2], Received Signal Strength Indicator (RSSI)based localisation [3], or, when not relying on radio-frequency (RF) signals, using Visible Light Positioning (VLP)-based localisation [4]. Due to the less stringent tracking accuracy demands for common indoor navigation applications, RSSIbased localisation systems are often being deployed, given their relatively low deployment cost and the compatibility with WiFi-enabled off-the-shelf devices, such as smartphones or tablets. Moreover, these allow an easy development of localization Apps that show the user's location and guide him or her to the desired destination via a graphical user interface. Currently, quite some research is being performed on the improvement of location tracking accuracy in RSSIbased systems. This paper aims to test and assess several of these approaches and quantify their impact: (i) applying path building (adding semantic data such as history of previous predictions in combination with a maximal speed and with the physical environment), (ii) processing multiple scans at one location using filtering techniques, (iii) accounting for the user's maximal speed in a dynamic way using a step counter, (iv) switching the frequency band from 2.4 to $5 \mathrm{GHz}$, and (v) changing the position of the mobile device with respect to the user's body.

In Section II, the main functionality of the App is briefly discussed. Section III presents the localisation algorithm with the different optimization strategies and Section IV describes the test environment and equipment. Section V discusses the results of the tests, and Section VI summarizes the main findings of this paper.

\section{MAIn FUnCTIONALITY OF THE APP}

The mobile application, using Android API 19 (KitKat), has been programmed to be fully responsive and extends an already existing application [5] which was based on a network planning tool [6]. This allows scaling on every device whilst keeping crucial information always visible. The App makes use of Android Managers, built-in packages that allow users to interact with the hardware of the device. Two important examples are the WiFiManager and SensorManager packages. The WiFiManager class allows performing a scan of the available WiFi access points (APs). The result is a list of AP names combined with their RSSI values. The SensorManager class is used to retrieve accelerometer data.

As will be discussed later, the main functionality of the App consists of estimating and visualising the user's location on the map of a ground floor. This is done via an algorithm that compares the measured RSSI values of all APs with RSSI values that are stored in a fingerprinting database. This database can be constructed either automatically, via the use of path loss prediction algorithms [7], or manually, through a fingerprinting procedure with the device at each location in the building. This procedure can be executed relatively fast, thanks to the smartphone's touchpad functionality which allows indicating the measurement location by a simple screen tap. Fingerprinting data can also be stored locally in an XML file. Other features of the App are related to usability and include multi-touch zooming, horizontal and vertical scrolling, or taking screenshots. Fig. 1 shows a screenshot of the 
graphical user interface of the location tracking App, with indication of the estimated current location (red dot on the far left).

\section{LOCALISATION ALGORITHM AND OPTIMIZATION STRATEGIES}

In the basic algorithm, the current location is estimated by only considering the most recent RSSI measurements from a set of APs. Based on the collected RSSI data, the most likely location is determined based on a calculation of sums of mean-square errors (sum of MSEs): i.e., the location of which the predicted RSSIs yield the lowest sum of MSEs compared to the measured RSSIs, is the most probable location. The predicted RSSIs are retrieved from the fingerprinting database. A full description of this algorithm can be found in [8].

\section{A. Optimization strategies}

1) Using path building: The basic algorithm is historyunaware, meaning that it does not account for previous locations. A path building approach exploits physical environment characteristics and a motion model as constraints to determine the most likely path (i.e., sequence of locations) instead of only the most likely instantaneous position. These two constraints ensure that no walls are crossed by the path (physical environment) and that no unrealistically large distances are traveled within a given time frame (motion model). The last position of the most likely path is then assumed to be the most likely current location [8]. The path calculations are not restarted every time a new measurement is received but the paths from a previous iteration serve as input for the current iteration along with the new measurements.

2) Averaging out multiple WiFi scans: As WiFi RSSI signals at a certain location often show large variations (due to the equipment, the body of the user carrying the mobile device [9], changes in the environment...), it can be expected that localisation accuracy will be severely impacted when the number of RSSI samples per AP per time instant is low. Therefore, it will be investigated to what extent the prediction accuracy is affected when the amount of collected RSSI scans at each location (and from each AP) is varied between 1 and 5 and the average value over the number of RSSI scans is used for further processing. Note that while path building uses information from previous locations (i.e., sample(s) from previous locations at previous time instants) to construct a path, this approach only uses information at the current location (i.e., multiple samples at current location at current time instant). Both approaches can be combined in this paper: path building will be deployed on top of the averaging approach.

3) Advanced averaging: Here, a more advanced averaging approach will be used, accounting for the reliability of the samples. To estimate the output RSSI values, all values corresponding to a certain location and time instant are being used in the calculation. In this approach, the following formula has been used to estimate the output RSSI value (instead of just averaging the scanned RSSI values):

$$
\operatorname{RSSI}_{\mathrm{i}}^{\text {pred }}=\alpha \cdot \operatorname{RSSI}_{\mathrm{i}}+(1-\alpha) \cdot \operatorname{RSSI}_{\mathrm{i}-1}^{\text {pred }},
$$

where $\operatorname{RSSI}_{\mathrm{i}}^{\text {pred }}$ is the output RSSI value after processing i scans at the considered location, RSSI $_{i}$ is the raw i'th sample or scan. i runs from 1 to the number of available RSSI samples/scans and RSSI $1=\mathrm{RSSI}_{1}$. The value $\alpha$ assigns more or less weight to the current scan, based on the estimated reliability of the sample. As reliability indicator, the RSSI difference between two subsequent samples is taken. A sudden large difference then indicates an unreliable sample and reduces the weight of the most recent sample (smaller $\alpha$ value). Table I shows the relationship between the RSSI difference and the $\alpha$ value. Note that this filter is separately applied to RSSI signals obtained from each of the APs.

TABLE I: Relationship between RSSI difference between two subsequent samples $\left(\Delta_{\mathrm{RSSI}}[\mathrm{dB}]=\mathrm{RSSI}_{\mathrm{i}}-\mathrm{RSSI}_{\mathrm{i}-1}\right)$ and $\alpha$ value in eq. (1)

\begin{tabular}{|r||r|r|r|r|r|r|r|}
\hline$\Delta_{\text {RSSI }}$ & $0-5$ & $6-10$ & $11-15$ & $16-25$ & $26-50$ & $51-75$ & $76-100$ \\
\hline$\alpha$ & 1 & 0.75 & 0.70 & 0.60 & 0.35 & 0.10 & 0.05 \\
\hline
\end{tabular}

4) Sensor fusion - using accelerometer data: All contemporary smartphones and tablets have an in-built accelerometer. The data obtained from it, can be used for localisation purposes, as it measures the acceleration of the device with respect to the gravity vector. Common approaches are deriving the velocity vector at each instant, detecting whether or not a user is moving, or deriving the number of steps taken by the user carrying the mobile device. In this paper, there will be focussed on the latter approach. Android has dedicated interfaces for step counting. In this paper, the number of steps will be combined with a fixed step length of $0.75 \mathrm{~m}$ (as observed from the test person) to estimate the user's maximal speed as used in the motion model of the path building approach. This allows accounting for a dynamically varying user velocity.

5) Switching frequency band: Generally, the $2.4 \mathrm{GHz}$ band is used for WiFi-based localisation, due to the better compatibility with most devices and the larger range of the access points. Thanks to the high density of wireless nodes in the testbed (see Section IV) and the $5 \mathrm{GHz}$-compatibility of both the testbed and the smartphone, it will be investigated if there is a difference in localisation accuracy when the $5 \mathrm{GHz}$ band is used.

6) Position of the mobile device with respect to the body: As the human body approaches the smartphone, the radiation pattern of the smartphone's antenna will be altered, resulting in unpredictable RSSI values. This effect was already quantified for small body-worn nodes in [9], and will here be tested for two positions of the smartphone relative to the body: close to the body (normal usage) and away from the body (stretched arms).

\section{TEST EQUIPMENT}

The experiments are conducted on a wireless testbed, located on the third floor of an office building in Ghent, covering 


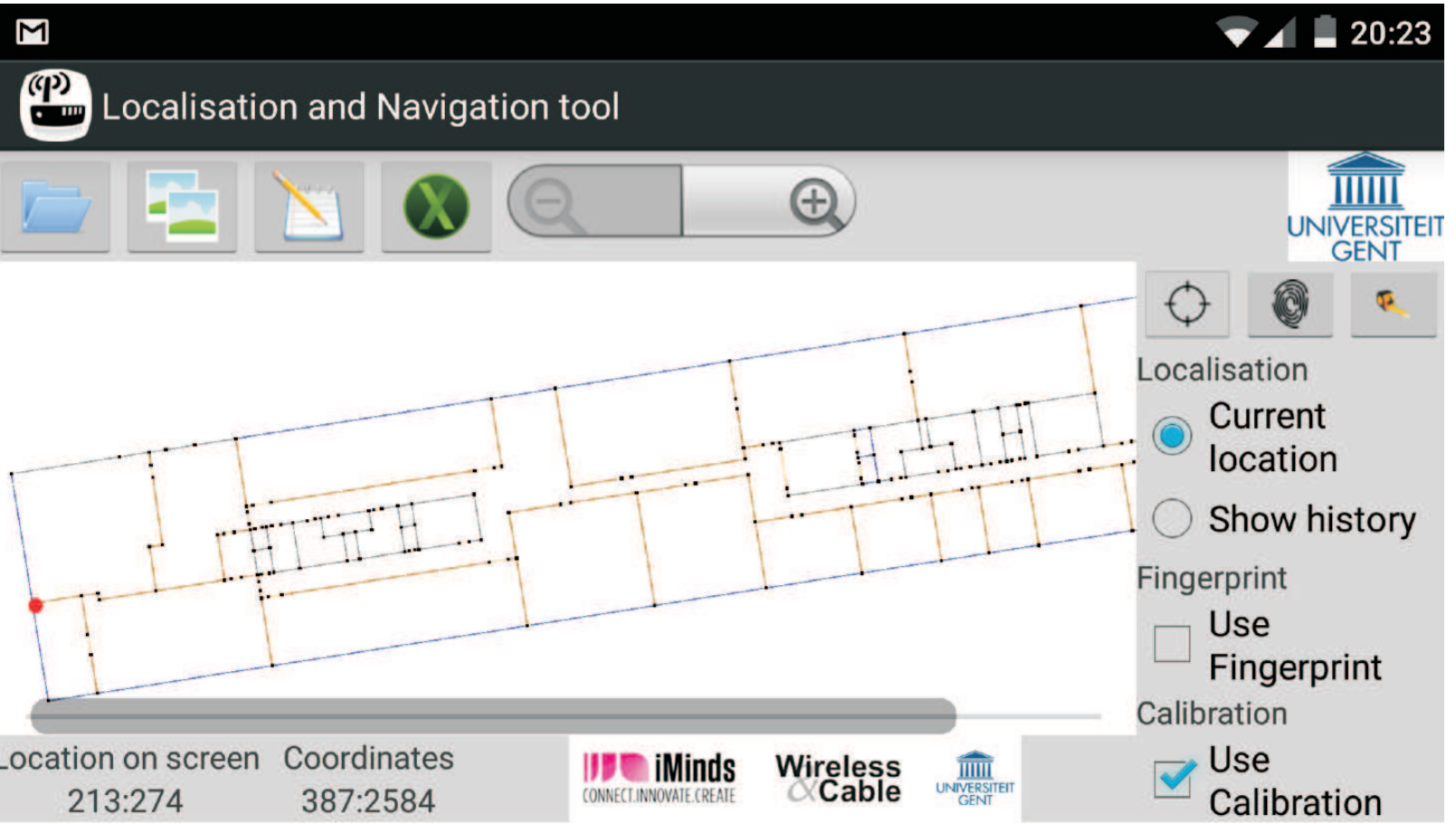

Fig. 1: Screenshot of the graphical user interface of the location tracking App with indication of estimated current location (red dot).

over $1500 \mathrm{~m}^{2}$ (17 $\mathrm{m}$ by $90 \mathrm{~m}$, see Figure 2$)$. The building floor is designed with light layered drywalls (orange walls in Figure 2) around a concrete core (grey walls in Figure 2). The testbed network consists of 48 fixed nodes at a height of $2.5 \mathrm{~m}$ (purple dots in Figure 2). In this work, the fixed testbed nodes operate in transmit node and all intelligence is available in the Android App (receiver). This allows a quick deployment of the App in other environments, as the App just scans the RSSI of the APs within its range. The smartphone that is used for all tests is a OnePlus 2 device with 4 GB RAM and Quad-core $1.82 \mathrm{GHz}$ Cortex-A57 processor.

Three test trajectories were chosen, one of which is displayed in red in Figure 2. For each of the tested configurations, all three test paths were travelled five times and resulting errors were averaged. The ground truth, i.e., the correct locations for comparison, is built by indicating the correct location on the map, each time a checkpoint (marked by squares in Figure 2) is passed. It should be noted that when the number of deployed fixed nodes is reduced, the resulting accuracy will also decrease [8].

\section{RESUlTS}

A first set of results is obtained from the reference configuration at $2.4 \mathrm{GHz}$, in which the amount of scans per location is set to 1 , the maximal user speed is $2 \mathrm{~m} / \mathrm{s}$, and the 10 strongest APs are considered. On average over the three test paths, an average localisation error of $5.3 \mathrm{~m}$ is obtained (see Table II, line 'number of scans = 1', column 'Reference, Averaging'). In the following, the different optimization strategies will be considered and their effects will be quantified.
TABLE II: Average localisation error [m] for the reference algorithm and for the path building algorithm, as a function of the number of collected scans per measurement location, with regular averaging of the scans and using an advanced averaging

\begin{tabular}{|r|r|r|r|r|}
\hline Localisation error [m] & \multicolumn{2}{|c|}{ Reference } & \multicolumn{2}{c|}{ Path building } \\
\hline number of scans & Averaging & Advanced & Averaging & Advanced \\
\hline $\mathbf{1}$ & 5.3 & 5.3 & 5.0 & 5.0 \\
\hline $\mathbf{2}$ & 4.8 & 4.5 & 4.6 & 4.3 \\
\hline $\mathbf{3}$ & 4.6 & 4.1 & 4.4 & 3.9 \\
\hline $\mathbf{4}$ & 4.1 & 3.9 & 3.8 & 3.7 \\
\hline $\mathbf{5}$ & 3.7 & 3.6 & 3.5 & 3.5 \\
\hline
\end{tabular}

\section{A. Using path building}

When path building is applied, meaning that the maximal velocity of the user is limited at (here) $2 \mathrm{~m} / \mathrm{s}$ and paths through walls are forbidden, the localisation error decreases to $5.0 \mathrm{~m}$, a slight improvement over the reference configuration (see Table II, line 'number of scans = 1', column 'Path building, Averaging').

\section{B. Averaging out multiple WiFi scans vs. 1 scan}

Table II shows the average localisation error when averaging out multiple WiFi scans per location (columns 'Averaging'). It shows that using multiple scans is an efficient way to reduce the localisation error: from $5.3 \mathrm{~m}$ to $3.7 \mathrm{~m}$ for the reference algorithm and from $5.0 \mathrm{~m}$ to $3.5 \mathrm{~m}$ for the path building algorithm. It is confirmed that the benefit of path building with respect to the reference algorithm is limited here (improvement of 0.2 to $0.3 \mathrm{~m}$ ). 


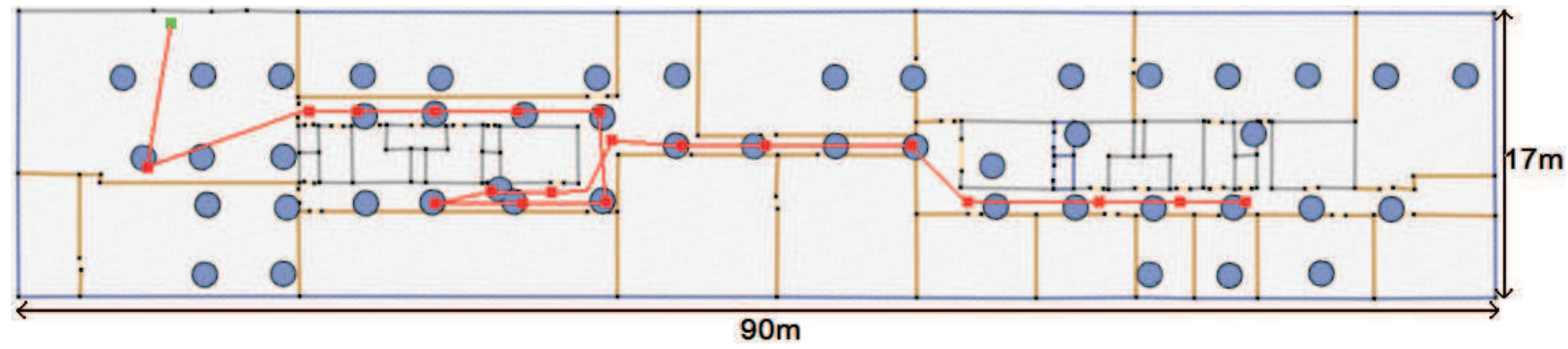

Fig. 2: Test environment $(90 \mathrm{~m} \times 17 \mathrm{~m})$ with indication of fixed testbed nodes (purple dots) and one of the three test trajectories (checkpoints are red squares, the green square indicates the first checkpoint).

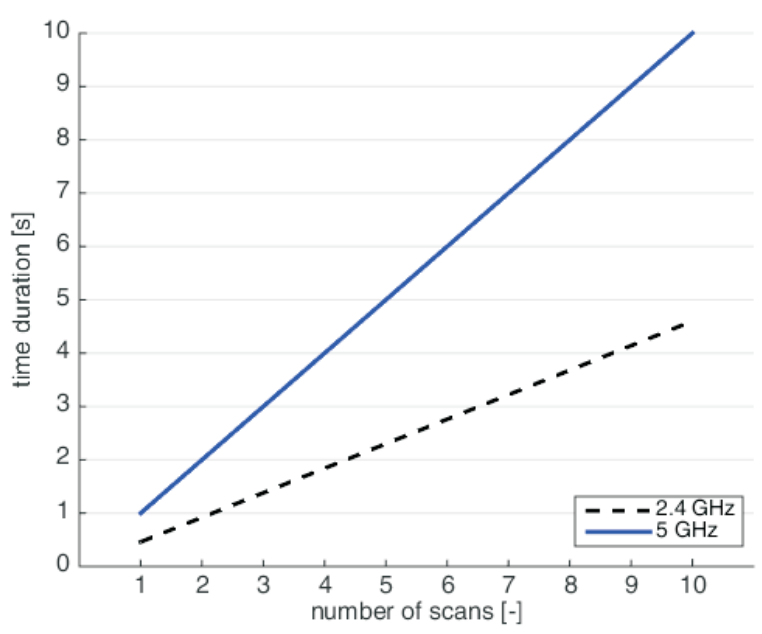

Fig. 3: Time required to perform WiFi scans in the $2.4 \mathrm{GHz}$ and $5 \mathrm{GHz}$ frequency band.

A limitation to increasing the number of WiFi scans, is the time required to perform these scans. Fig. 3 shows the time that is required to perform a number of passive scans for the two frequency bands that are considered here. Scanning the $5 \mathrm{GHz}$ band takes longer than the $2.4 \mathrm{GHz}$ band, since it contains more channels. For 10 scans at $2.4 \mathrm{GHz}$, up to $4.5 \mathrm{~s}$ are needed, and even up to $10 \mathrm{~s}$ at $5 \mathrm{GHz}$. It should be noted that for real-time location tracking, this time duration needs to be limited to a few seconds. An faster alternative is to have the App perform an active scan on the correct frequency channel, but unfortunately, this functionality is banned in the most recent Android APIs.

\section{Applying advanced averaging vs. regular averaging}

Table II shows the average localisation error when applying an advanced averaging technique on multiple WiFi scans (columns 'Advanced'). It shows that not only inputting multiple scans to the filter is beneficial, but also that applying the advanced averaging yields better results than regular averaging (improvement up to $0.5 \mathrm{~m}$, for path building algorithm combined with 3 scans per location). It is again confirmed that there is a small benefit when using path building (improvement of 0.1 to $0.3 \mathrm{~m}$ compared to the reference algorithm). Figure 4 shows the ground truth (black path) with the reconstructed paths obtained by regular averaging (green) and by applying the advanced averaging (red). It shows that advanced averaging indeed slightly outperforms regular averaging.

\section{Sensor fusion - using accelerometer data vs. fixed speed}

When using the accelerometer data to derive the actual speed of the person, a more accurate grid of next possible locations can be built. Unlike the fixed maximal speed of $2 \mathrm{~m} / \mathrm{s}$ used before, this maximal speed (and the corresponding maximal movement per time unit) is now dynamic. Table III shows that a significant improvement is obtained using the accelerometer-derived maximal speed: up to $37 \%$ (from $3.5 \mathrm{~m}$ to $2.2 \mathrm{~m}$ ) for 5 scans. Again, the advanced averaging technique performs slightly better than the regular averaging approach, but gains are limited to $0.1 \mathrm{~m}$.

TABLE III: Average localisation error [m] for the path building algorithm, for a fixed maximal speed ('fixed') and for a dynamically determined maximal speed using accelerometer data (sensor fusion, 'dynamic') as a function of the number of collected scans per location, for regular averaging and for advanced averaging

\begin{tabular}{|r|r|r|r|r|}
\hline \multicolumn{1}{|c|}{ Localisation error [m] } & \multicolumn{2}{|c|}{ Fixed } & \multicolumn{2}{c|}{ Dynamic } \\
\hline \multicolumn{1}{c|}{ number of scans } & Averaging & Advanced & Averaging & Advanced \\
\hline $\mathbf{1}$ & 5.0 & 5.0 & 2.8 & 2.8 \\
\hline $\mathbf{2}$ & 4.6 & 4.3 & 2.7 & 2.6 \\
\hline $\mathbf{3}$ & 4.4 & 3.9 & 2.5 & 2.4 \\
\hline $\mathbf{4}$ & 3.8 & 3.7 & 2.5 & 2.4 \\
\hline $\mathbf{5}$ & 3.5 & 3.5 & 2.2 & 2.2 \\
\hline
\end{tabular}

\section{E. $5 \mathrm{GHz}$ frequency band vs. $2.4 \mathrm{GHz}$ band}

Table IV shows the localisation error for the $2.4 \mathrm{GHz}$ and $5 \mathrm{GHz}$ band, with advanced averaging, using path building and an accelerometer-based speed detection. The table shows that there is a small advantage in using the $5 \mathrm{GHz}$ band. However, Fig. 3 shows that it takes longer to scan the $5 \mathrm{GHz}$ band, which impacts the real-time tracking capabilities.

\section{F. Position of the mobile device with respect to the body}

Traveling the three test paths showed that holding the smartphone away from the body leads to lower localisation 


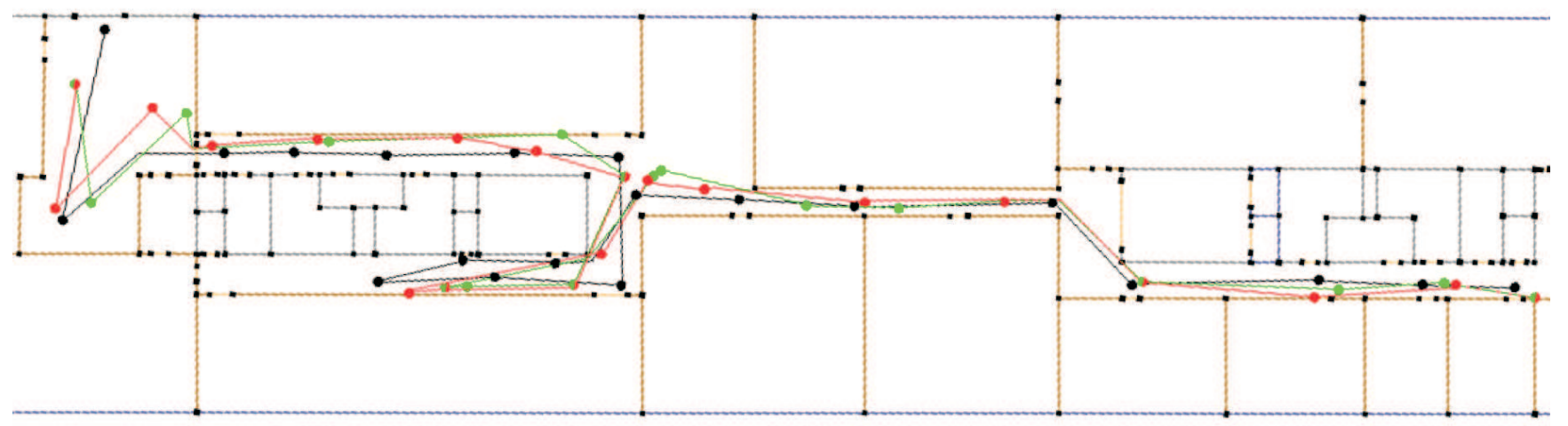

Fig. 4: Comparison of the reconstructed paths when averaging the RSSI scans (green) vs. applying advanced averaging (red). The black path is the ground truth.

TABLE IV: Average localisation error [m] for the path building algorithm with a dynamically determined maximal speed and advanced averaging, as a function of the number of collected scans per location, for the $2.4 \mathrm{GHz}$ and $5 \mathrm{GHz}$ frequency band

\begin{tabular}{|r||r|r|r|r|r|}
\hline \multicolumn{1}{|c||}{ Localisation error [m] } & \multicolumn{5}{|c|}{ number of scans } \\
\hline frequency band & $\mathbf{1}$ & $\mathbf{2}$ & $\mathbf{3}$ & $\mathbf{4}$ & $\mathbf{5}$ \\
\hline $\mathbf{2 . 4} \mathbf{~ G H z}$ & 2.8 & 2.6 & 2.4 & 2.4 & 2.2 \\
\hline $\mathbf{5 ~ G H z}$ & 2.7 & 2.5 & 2.2 & 2.2 & 1.9 \\
\hline
\end{tabular}

errors than holding it close to the body: the errors close to the body equal $3.4 \mathrm{~m}$ (horizontally) or $2.96 \mathrm{~m}$ (vertically), while errors away from the body equal $2.3 \mathrm{~m}$ (horizontally) or $2.1 \mathrm{~m}$ (vertically). The tests were performed at $5 \mathrm{GHz}$, using path building, advanced averaging, a dynamic speed detection, and using 5 scans per location. This shows that body compensation algorithms [9] can indeed be useful. A vertical position of the phone performs slightly better than the horizontal position, due to the position of the antenna inside the device (near the top side).

\section{CONCLUSIONS}

In this paper, different optimization strategies for a location tracking App are investigated and quantified when used on an Android smartphone. A basic RSSI approach yields average prediction errors just above $5 \mathrm{~m}$ over a set of three test trajectories in an office building. Application of path building approaches reduces this error to less than 5 meter. Using 5 RSSI samples per location and processing them with an advanced averaging technique further reduces the average prediction error to $3.5 \mathrm{~m}$. The largest improvement $(-37 \%$, to $2.2 \mathrm{~m}$ ) is obtained by adding a step counter to the algorithm for limiting the maximal speed of the user. Finally, another improvement is obtained when the access point network is configured to operate at $5 \mathrm{GHz}$ instead of at $2.4 \mathrm{GHz}$. Combining all optimal settings mentioned above yields an average error of $1.9 \mathrm{~m}$. In the future, the strategies will be tested in other environments and a comparison of the current WiFi-approach will be made with a Bluetooth Low Energy (BLE)-based approach. The location tracking algorithm will be extended with a navigation feature by converting the map to a graph and applying Dijkstra's algorithm between the predicted location and the destination location.

\section{REFERENCES}

[1] D. Dardari, A. Conti, U. Ferner, A. Giorgetti, and M. Z. Win, "Ranging with ultrawide bandwidth signals in multipath environments," Proceedings of the IEEE, vol. 97, no. 2, pp. 404-426, 2009.

[2] E. Elnahrawy, J. Austen-Francisco, and R. P. Martin, "Adding angle of arrival modality to basic rss location management techniques," in Wireless Pervasive Computing, 2007. ISWPC07. 2nd International Symposium on. IEEE, 2007.

[3] A. W. S. Au, C. Feng, S. Valaee, S. Reyes, S. Sorour, S. N. Markowitz, D. Gold, K. Gordon, and M. Eizenman, "Indoor tracking and navigation using received signal strength and compressive sensing on a mobile device," IEEE Transactions on Mobile Computing, vol. 12, no. 10, p. 20502062, 2013.

[4] J. Armstrong, Y. A. Sekercioglu, and A. Neild, "Visible light positioning: A roadmap for international standardization," IEEE Communications Magazine, vol. 51, no. 12, pp. 68-73, December 2013.

[5] D. Plets, R. Mangelschots, K. Vanhecke, L. Martens, W. Joseph, "A Mobile App for Real-Time Testing of Path-Loss Models and Optimization of Network Planning," in 27th Annual IEEE International Symposium on Personal, Indoor and Mobile Radio Communications, Valencia, Spain, 4-7 September 2016, paper no. 1570250866.

[6] D. Plets, W. Joseph, K. Vanhecke, E. Tanghe, and L. Martens, "Simple indoor path loss prediction algorithm and validation in living lab setting," Wireless Personal Communications, pp. 1-18, 10.1007/s11277-011-04674. [Online]. Available: http://dx.doi.org/10.1007/s11277-011-0467-4

[7] - "Coverage Prediction and Optimization Algorithms for Indoor Environments," EURASIP Journal on Wireless Communications and Networking, Special Issue on Radio Propagation, Channel Modeling, and Wireless, Channel Simulation Tools for Heterogeneous Networking Evaluation, vol. 1, 2012. [Online]. Available: http://jwcn.eurasipjournals.com/content/2012/1/123

[8] J. Trogh, D. Plets, L. Martens, and W. Joseph, "Advanced real-time indoor tracking based on the viterbi algorithm and semantic data," International Journal of Distributed Sensor Networks, vol. 11, no. 10, 2015. [Online]. Available: http://dsn.sagepub.com/content/11/10/271818.abstract

[9] J. Trogh, D. Plets, A. Thielens, L. Martens, and W. Joseph, "Enhanced indoor location tracking through body shadowing compensation," IEEE Sensors Journal, vol. 16, no. 7, pp. 2105-2114, April 2016. 